Cohort study

\section{Aiming too high or too low? Searching for the appropriate therapeutic thresholds in hypertension is not over yet}

10.1136/ebmed-2014-110104

\section{Artur Fedorowski}

Department of Clinical Sciences in Malmö, Lund University, Malmö, Sweden

Correspondence to: Professor Artur Fedorowski, Department of Cardiology, Skåne University Hospital, Inga Marie Nilssons gata 46, Malmö 20502, Sweden; artur.fedorowski@med.lu.se

Commentary on: Rodriguez CJ, Swett K, Agarwal SK, et al. Systolic blood pressure levels among adults with hypertension and incident cardiovascular events: the Atherosclerosis Risk in Communities Study. JAMA Intern Med 2014;174:1252-61.

\section{Context}

Increased arterial blood pressure (BP) is an established and modifiable cardiovascular (CV) risk factor. Consequently, the decision on antihypertensive treatment has been in the focus of changing recommendations over the past decades, initially with higher accepted BP thresholds than those recommended by modern guidelines. However, recently published consensus statements have indicated that treatment goals should be less ambitious, as 'the lower the better' might not be applicable for ages $>60$ years and in uncomplicated hypertension. ${ }^{1}{ }^{2}$ Moreover, the traditional definition of increased BP that warrants pharmacological treatment has been questioned since studies have suggested that elevated night-time BP, but not daily, office or self-measured BP, is a better marker of increased CV risk. ${ }^{3}$ In this population-based study, the authors examined incidence of $\mathrm{CV}$ disease among hypertensive participants stratified according to baseline systolic BP (SBP) with the lowest SBP stratum as a reference.

\section{Methods}

In the prospective cohort of the Atherosclerosis Risk in Communities Study, participants who met the hypertension criteria at baseline (sitting BP >140/90 mm Hg, or history of hypertension), without history of CV disease were identified. The participants were classified as low (reference, $<120 \mathrm{~mm} \mathrm{Hg}$ ), standard (120-139 mm Hg) and elevated (>139 $\mathrm{mm} \mathrm{Hg}$ ) SBP, and, additionally, as those below and above $160 \mathrm{~mm} \mathrm{Hg}$. The primary outcome was a composite CV event of myocardial infarction, CV death, heart failure and ischaemic stroke. The authors clearly described the selection process and how the CV end points during follow-up were assessed. The main results were reported as HRs with 95\% CIs.

\section{Findings}

A cohort of 5466 hypertensive participants (women, 55.9\%; age, 55.5 \pm 5.7 years) was followed up for about 22 years. During this period, a total of 1622 CV events occurred. Among participants with low (22.7\%), normal (33.6\%) and elevated SBP (43.7\% of all), proportions of those treated with antihypertensive drugs at baseline were 99.4\%, 89.8\% and 46.1\%, respectively. In the fully adjusted model, the elevated SBP conferred higher risk of incident CV disease (HR 1.46; 95\% CI 1.26 to 1.69), whereas there was no difference between low and normal SBP (HR 1.00; 95\% CI 0.85 to 1.17). Moreover, the age-adjusted risk of incident CV disease was distinctly higher among participants with SBP $\geq 160 \mathrm{~mm} \mathrm{Hg}$ (HR 1.73; 95\% CI 1.46 to 2.05) than in those with SBP $140-159 \mathrm{~mm} \mathrm{Hg}$ (HR 1.16; 95\% CI 1.01 to 1.34$)$.

\section{Commentary}

This observational study emphasises the importance of identifying those hypertensive patients who have the highest probability to benefit from pharmacological intervention. It also offers a strong argument in the discussion about the higher treatment goals in uncomplicated hypertension. However, the main finding was not sufficiently highlighted by the authors. The clinically important increase in CV risk was observed only in a minor subset of patients with baseline SBP above $160 \mathrm{~mm} \mathrm{Hg}$. This group constituted approximately 25\% of all participants with SBP $>140 \mathrm{~mm} \mathrm{Hg}$ who were, apart from that, less frequently treated as compared with the low-normal SBP group. It should be noted that the authors did not report the incidence of major CV events in normotensive participants, so the potential impact of hypertension with a satisfactory BP control on the development of CV disease could not be evaluated. In summary, when properly treated to the SBP threshold below $160 \mathrm{~mm} \mathrm{Hg}$, $\mathrm{CV}$ risk associated with uncomplicated hypertension did not substantially differ across increasing strata of office SBP. In contrast, hypertensive patients with untreated or treated baseline SBP above $160 \mathrm{~mm} \mathrm{Hg}$ demonstrated significantly higher CV event incidence.

\section{Implications for practice}

This study provides a strong argument that former treatment goals in uncomplicated hypertension among middle-aged individuals should be re-evaluated. The possible advantage of lowering SBP below not only $120 \mathrm{~mm} \mathrm{Hg}$ but also below $140 \mathrm{~mm} \mathrm{Hg}$ in preventing CV disease has been questioned. Thus, the most recent recommendations for the elderly, suggesting SBP levels $<150 \mathrm{~mm} \mathrm{Hg}$ as the realistic treatment goal, can be extrapolated onto middle-aged individuals free from $\mathrm{CV}$ disease, that is, those with low-to-moderate CV risk. Moreover, the decision on whether or not to treat the increased office SBP above $160 \mathrm{~mm} \mathrm{Hg}$ may be additionally supported by elevated night-time BP.

\section{Competing interests None.}

Ethics approval University of North Carolina.

Provenance and peer review Commissioned; internally peer reviewed.

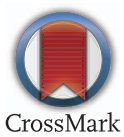

\section{References}

1. James PA, Oparil S, Carter BL, et al. 2014 evidence-based guideline for the management of high blood pressure in adults: report from the panel members appointed to the Eighth Joint National Committee (JNC 8). JAMA 2014;311: 507-20.

2. Mancia G, Fagard R, Narkiewicz K, et al. 2013 ESH/ESC guidelines for the management of arterial hypertension: the Task Force for the Management of Arterial Hypertension of the European Society of Hypertension (ESH) and of the European Society of Cardiology (ESC). Eur Heart J 2013;34:2159-219.

3. Hermida RC, Ayala DE, Mojon A, et al. Decreasing sleep-time blood pressure determined by ambulatory monitoring reduces cardiovascular risk. J Am Coll Cardiol 2011;58:1165-73. 